\title{
Transformative Bildung als praktizierte Solidarität angesichts globaler Krisen
}

\author{
Norbert Mette \\ Technische Universität Dortmund
}

Kontakt: norbert.mette@freenet.de

eingereicht: 03.02.2021; überarbeitet: 30.03.2021; angenommen: 16.05.2021

\begin{abstract}
Zusammenfassung: „Transformative Bildung“ ist keine Aufgabe, die sich erst angesichts der aktuellen krisenhaften Weltlage für Erziehung und Bildung ergibt. Sondern pädagogisches Handeln hat es grundlegend mit einer transformatorisch sich vollziehenden Entwicklung der Individuen zu tun. Ein solcher intergenerationeller Lernprozess steht je neu vor der Prüfung, ob der gesellschaftliche Kontext, wie er ist, zukunftsfähige Lebensmöglichkeiten für alle bereit hält.

Schlagwörter: Globale Krisen, Bildung, Kreativität, Freiheit, Solidarität

Abstract: Transformational education is not only a challenge because of the recent critical global situation. Pedagogical practice has fundamentally to do with a transformational development of the individuals. This intergenerational process of learning has to consider each newly if the context of the present society provides sustainable opportunities of life for all, especially for the next generations.

Keywords: global crises, education, creativity, freedom, solidarity
\end{abstract}

„Daß es ,so weiter' geht, ist die Katastrophe“ (Benjamin, 1991, S. 683). Mit Blick auf die Weltlage ist dieses Zitat von Walter Benjamin (1892-1940) aus dem Jahr 1937 höchst aktuell. Besonders betroffen sind von der sich weiter beschleunigenden katastrophalen Entwicklung (soziale Spaltung, Klimakrise, Raubbau an der Natur, „Modernisierung“ der Waffen zur Kriegsführung u. a. m.) die kommenden Generationen mitsamt der Fauna und Flora und bereits jetzt schon die vom Wohlstand der Wenigen ausgeschlossene Mehrheit der Weltbevölkerung. Um nichts weniger als des Überlebens willen ist eine tiefgreifende Veränderung in so gut wie allen Bereichen des Zusammenlebens angesagt. Politik und Wirtschaft tragen dafür eine maßgebliche Verantwortung.

Aber auch Erziehung und Bildung sind gefordert, damit es zu einem entsprechenden Bewusstsein kommt. Es bedarf, wie Klaus Seitz zu Recht fordert, einer umfassenden Bildungswende (Seitz, 2017, S. 166; auch Lang-Wijtasik \& Erichsen-Morgenstern, 2019; Scheunpflug, 2019), auch in der Religionspädagogik (Bederna, 2019; Gärtner, 2020; ÖRF, 2020). Zur vertiefenden Klärung des Verhältnisses von Erziehung/Bildung und Transformation sollen im Folgenden drei Autoren - Helmut Peukert (1), Paulo Freire (2) und Papst Franziskus (3) - herangezogen werden, die je auf ihre Weise darauf verweisen, dass dem Prozess der Erziehung und Bildung selbst, radikal verstanden, bereits eine transformierende Dynamik innewohnt, dass also das Reden von "transformativer Bildung“ keineswegs bloß eine „von außen“ herangetragene, den aktuellen Herausforderungen geschuldete Angelegenheit ist.

Gemeinsam ist übrigens den drei genannten Autoren der jeweils von ihnen eingenommene Ausgangspunkt, nämlich „,an die Ränder des Daseins gehen, um die Welt so zu sehen, wie sie ist" (Papst Franziskus, 2020b, S. 19). 


\section{Intersubjektive Kreativität (Helmut Peukert)}

Das pädagogische EEuvre von Helmut Peukert $\left({ }^{*} 1934\right)$ kreist zentral um den Begriff „Transformation“ (Peukert, 2015). Den Ausgangspunkt dafür bildet folgende Überlegung: In Erziehung und Bildung geht es darum, der nächsten Generation ein Leben auf Zukunft hin zu ermöglichen. Die Zeitspanne verlängert sich noch weiter nach vorn, wenn man an die Ausbildung von zukünftigen Lehrerinnen und Lehrern denkt. Sie werden es in ihrer dreißig- bis vierzigjährigen Dienstzeit mit Heranwachsenden zu tun haben, die weit bis ins 22. Jahrhundert hinein leben werden - vorausgesetzt, dass auch für diese übernächste Generation und die ihr folgenden noch die notwendigen Lebensgrundlagen vorhanden sind. Dass genau das keineswegs so selbstverständlich erscheint, wie es früher der Fall gewesen sein mag, stellt für Erziehung und Bildung eine neuartige, dermaßen drastisch bisher nicht gegebene Herausforderung dar.

Die künftigen Generationen werden in ihrer Lebenszeit aufgrund radikaler globaler Veränderungen vor Aufgaben gestellt sein wie noch keine Generation zuvor - wobei auch schon in den letzten zweihundert Jahren ein gewaltig sich beschleunigender Wandel zu verarbeiten gewesen ist. Aber folgt man sozialwissenschaftlichen Analysen, so Helmut Peukert, hat sich die Lage gegenwärtig nochmals verschärft (dazu auch WBGU-Bericht, 2011). Um es an den Bereichen Wirtschaft, Politik und Kultur zu verdeutlichen:

„Das ökonomische System, das der Produktion und Verteilung von Gütern zur Befriedigung von Bedürfnissen und damit zur Sicherung des Lebens auf einem bestimmten Niveau dienen soll, droht die Biosphäre insgesamt und damit das Leben in den bisher bekannten Formen überhaupt zu zerstören. Das System der internationalen Politik, in dem die eigene Existenz durch die Gewißheit der bleibenden Möglichkeiten gegenseitiger Vernichtung militärisch gesichert werden soll, wird instabiler und droht über die Existenz der Konkurrenten hinaus die Existenz der menschlichen Gattung als ganzer zu vernichten. Die kulturellen Modernisierungsprozesse haben traditionelle Lebensformen aufgelöst, aber von sich aus kaum integrierende Grundorientierungen hervorgebracht; das kulturelle System ist schwerlich in der Lage, diese Erfahrungen aufzufangen, vielmehr wird seine Tendenz, fragmentiertes Bewußtsein zu produzieren, noch gefördert" (Peukert, 2015, S. 325).

Von diesen von Peukert als selbstdestruktiv gekennzeichneten Tendenzen ist die südliche Hemisphäre bereits jetzt besonders nachhaltig betroffen. Doch die Spaltung in Arm und Reich, in Privilegierte und Benachteiligte hat längst auch in die „hoch entwickelten“ Bereiche unseres Planeten übergegriffen und vertieft sich im globalen Ausmaß immer mehr. Die Zukunftsaussichten sind bereits für die nächste Generation - und das ist einem Teil der gegenwärtig Heranwachsenden durchaus bewusst - höchst prekär. Das bedeutet: Wo die Zukunft der Welt dermaßen in Frage steht, „wird der Vorgang von Erziehung selbstwidersprüchlich: was zukünftiges Leben ermöglichen soll, gefährdet dieses Leben“ (Peukert, 2015, S. 298).

Sicher, so könnte man einwenden, wird manches dafür getan, um es nicht zum „endgültigen Knall“ kommen zu lassen. Verwiesen wird gern etwa auf die Bemühungen, es seitens Politik und Wirtschaft mithilfe eines "green deal“ zum Stoppen des Klimawandels kommen oder durch multi- oder internationale Verhandlungen die jeweils neu entflammten Brennpunkte der Erde nicht zum Flächenbrand werden zu lassen. Mit der Digitalisierung wird eine zukunftsträchtige Umgestaltung aller Lebensbereiche erhofft und vorangetrieben $u$. a. m. Aber auch eher rückwärts gerichtete Reaktionen sind zu verzeichnen, die zu einem guten Teil aus einer Mischung von Angst und Wut vor der Folgen der eingetretenen globalen Situation herrühren: Ein neuer Nationalismus, unterstützt von populistischen Kräften, macht sich breit; die Politikverdrossenheit nimmt zu; autoritäre Regime werden hoffähig; das Wettrüsten und die Entwicklung immer präziser wirkender Waffensysteme sind erneut im vollen Gange u. s. w. Ein Denken und Handeln nach der Devise, zuerst für sich selbst zu sorgen ohne Rücksicht auf andere, ist 
weithin tonangebend geworden - oder auch, sich einfach in die eigenen vier Wände zurückzuziehen, weil sich sowieso nichts ändern lasse. Die „neue Unübersichtlichkeit“, wie Jürgen Habermas 1985 die damalige gesellschaftliche Lage charakterisiert hat, hat mit Blick auf heute wohl eher zugenommen.

Appelle zu einem notwendig gewordenen tiefgreifenden Bewusstseinswandel gibt es zuhauf. Die Frage ist nur, wie das entsprechende Bewusstsein beschaffen sein müsste und wie es zu erlangen ist. Dass den sich angesichts der Krisen stellenden Herausforderungen nicht mit von Angst geleiteten irrationalen oder kleinkarierten Reaktionen wirkungsvoll zu begegnen ist, liegt auf der Hand. Aber auch Maßnahmen wie "green deal“ oder Digitalisierung müssen sich kritisch befragen lassen, ob sie mit den von ihnen in Anschlag gebrachten Verfahren wirklich das zu erreichen vermögen, was sie beabsichtigen. Welche Rationalität liegt ihnen zugrunde?

Um das zu beantworten, muss zurückgefragt werden, welche Kräfte maßgeblich dafür ausschlaggebend waren bzw. sind, dass es zu der kurz angedeuteten krisenhaften Zuspitzung der Weltlage gekommen ist. Peukert sieht die Ursache darin, dass seit der Neuzeit eine bestimmte Form der Rationalität und ein davon geleitetes Handeln dominant geworden sind. Am deutlichsten lässt sich das an den Naturwissenschaften aufzeigen: Mithilfe von Experimenten wird versucht, die der Natur innewohnenden Gesetzmäßigkeiten zu entdecken. Das so gewonnene Wissen wird dazu genutzt, gezielter in die Abläufe der Natur eingreifen und über sie verfügen zu können. Dass auf diese Weise technische Errungenschaften gewonnen worden sind, die für die Menschen bis in ihren Alltag hinein vieles erleichtert und manches überhaupt erst ermöglicht haben (z. B. im Gesundheitsbereich), ist unbestreitbar. Aber je weiter und feiner dieses Wissen vorangetrieben wird, desto stärker werden auch die Schattenseiten beispielsweise in Form von nicht vorhergesehenen schädlichen Nebenwirkungen erkannt, die mit manchem neu gewonnenen technischen Know-how einhergehen oder aus ihm resultieren. Zudem lässt sich nicht länger übersehen, dass die natürlichen Ressourcen nicht unbegrenzt sind.

Von der auf Erweiterung und Steigerung der Verfügungsmöglichkeiten ausgerichteten Rationalität wird auch eine Verbesserung der Regelungen im sozialen Bereich erwartet. Mithilfe der an die Naturwissenschaften angelehnten instrumentell-experimentellen Vernunft wird alles Mögliche versucht, um die Abläufe vor allem in der Wirtschaft sowie auch in der Politik bis in den Wissenschafts- und Kulturbereich hinein effizienter zu gestalten und Störfaktoren auszuschalten. Fortschritt und Wachstum sind die leitenden Prinzipien, denen alles andere untergeordnet wird. Erfolg hat, wer sich im unerbittlich geführten Konkurrenzkampf schließlich durchsetzt. Wer dabei nicht mithalten kann, hat das Nachsehen und muss schauen, wie er oder sie damit zurechtkommt.

Die Auswirkungen dieses Denkens und Tuns bekommt nicht zuletzt der Erziehungs- und Bildungsbereich enorm zu spüren. Richtet sich auf ihn doch die Erwartung - und das wird durch die Ökonomisierung dieses Bereichs zusätzlich zu sichern versucht -, dass die jeweils heranwachsende Generation so sozialisiert wird, dass sie sich möglichst reibungslos in den gesellschaftlichen Mainstream einfügt und sich seine Normen zu eigen macht. Bildung wird zur Anpassung degradiert. Für die Bewältigung etwaiger aufkommender persönlicher Krisen, die die Funktionstüchtigkeit der Betroffenen beeinträchtigen, sind dafür vorgesehene therapeutische Einrichtungen zuständig, u. a. auch die Religion.

Was aber ist, wenn es sich bei den Krisen nicht bloß um Einzelfälle handelt, sondern wenn der Eindruck entsteht, dass die gesellschaftliche Entwicklung insgesamt einen immer manifester werdenden krisenhaften Verlauf nimmt - bis hin, wie angedeutet, zu ihrer möglichen Selbstdestruktion - und sich genau das bis in die innere Verfassung der Menschen hinein auswirkt? Wie erwähnt, ist das nach Peukert darauf zurückzuführen, dass die hier grob umrissene Rationalität, deren inneres Prinzip die Steigerung von Macht im Sinne von Verfügungsmöglichkeiten über eine zum Objekt manipulierte Wirklichkeit ist, absolute Geltung beansprucht. In detaillierten Analysen hat er jedoch aufgewiesen, dass dieser Anspruch nicht haltbar ist, sondern dass diese Rationalität selbstwidersprüchlich wird (dazu ausführlich 
Peukert, 2009). Das kann hier nicht im Einzelnen nachgezeichnet werden. Bei der auf der Logik der Machtsteigerung basierenden Rationalität - so jedenfalls ein wichtiges Ergebnis dieser Analysen - handelt es sich um eine bestimmte Ausprägung der Vernunft in Gefolge der neuzeitlichen Aufklärung. Neben ihr und vom Ansatz her gegen sie ist ein anderes und radikaleres Verständnis von Aufklärung auszumachen, das auf die Existenzerhellung des Menschen abzielt und daraus Folgerungen für eine zutiefst humane Lebensform abzuleiten und zu gestalten bemüht ist. Während in der einen Variante mit der Umwelt so umgegangen wird, dass sie vom Menschen zum Objekt gemacht wird mit dem Ziel, sie besser für sich ausnutzen zu können, geht es der anderen Variante darum, gerade diese Verdinglichung der Wirklichkeit und der daraus resultierenden Entfremdung des Menschen zu durchbrechen, indem in Anbetracht des Faktums der Endlichkeit nach den Bedingungen der Möglichkeit eines selbstbestimmten Existieren-Könnens in Freiheit gefragt wird. "Menschliche Existenz", so schreibt Peukert, „Subjektsein, ,Ich' sagen zu können, bedeutet die Möglichkeit, sich zur eigenen Existenz als alltägliches In-der-Welt-Sein in ein Verhältnis zu setzen und dieses In-ein-Verhältnis-Setzen nicht von anderen bestimmen zu lassen, sondern es selbst zu wählen“ (2015, S. 135). Dabei ist vor sich selbst und vor anderen für diese Wahl verantwortlich einzustehen. Die so gewonnene Freiheit verwirklicht sich allerdings erst voll in der Anerkennung anderer Freiheit. Damit ergibt sich aus der transzendentalen Analyse ein normatives Moment, nämlich dass die wechselseitige Anerkennung der Freiheit ein ethischer Grundakt ist, den zu vollziehen die Würde menschlichen Handelns ausmacht (Peukert, 2015, S. 302).

Wenn man nun nicht bloß nach der möglichen Freiheit, sondern nach der realen Freiheit fragt, zeigt sich, dass diese alles andere als ohne Weiteres gegeben, sondern in ihrer Verwirklichung auf vielfältige Weise prekär und bedroht ist - bis hin dazu, dass sie verwehrt wird. Von daher ist gemeinsam mit den anderen für sie zu kämpfen, um freie Lebensmöglichkeiten zu schaffen und zu sichern. Diese auf kommunikativer Vernunft basierende Lebensform besteht nach Peukert in einer intersubjektiven Kreativität, die „dem Willen zur Selbstbehauptung durch Macht entgegengesetzt" (Peukert, 2015, S. 303) sei worin ihre Verletzlichkeit bestehe.

Eine solche Ethik intersubjektiver Solidarität, die darauf zielt, mögliche Freiheit real werden zu lassen, bildet für ihn auch für pädagogisches Handeln die normative Grundlage. Geht es diesem doch darum, einem Heranwachsenden „erst die Möglichkeit zu schaffen, daß er in Freiheit mir [d. h. dem Erzieher, $\mathrm{NM}$ ] zustimmen oder widersprechen kann. Eine pädagogische Ethik will nicht nur Konsensmöglichkeiten für vorgetragene Ansprüche prüfen, sondern dazu verhelfen, Ansprüche überhaupt erst selbständig artikulieren zu können. Sie will denen, die noch nicht oder - weil sie zu verstört sind - nicht mehr reden können, erst zum Wort verhelfen [...] Die freie Anerkennung des anderen in seiner Freiheit bedeutet zu wollen, daß er im Modus intersubjektiv reflektierter Selbstbestimmung er selbst werden soll. Ein normativ dimensioniertes pädagogisches Handeln zielt auf die Genese von Subjekten, sie will Leben ermöglichen und weiß sich verantwortlich für dessen Vorbedingungen“ (Peukert, 2015, S. 303).

Mit dieser Ethik werden dem Erziehungsprozess keine fremden Normen eingetragen, sondern sie hat ihren Rückhalt in seiner Struktur selbst, die eben als solche transformatorisch ist, wie Peukert es exemplarisch anhand des Spracherwerbs von Kindern und der Entwicklung des moralischen Urteils darlegt. Zum einen habe Schleiermacher nachgezeichnet, wie Kinder im Zuge ihres Erlernens der eigenen Sprache und des Verstehens von mündlichen sowie schriftlichen Texten schöpferisch tätig werden (Peukert, 2015, S. 210f., S. 301f.). Zum anderen lasse sich den psychologischen Entwicklungstheorien zufolge die menschliche Entwicklung als ein dialektischer Prozess verstehen, in dem Phasen der Stabilität im Umgang mit der gegebenen Wirklichkeit aufgrund äußerer Einflüsse und innerer Vorgänge jeweils in eine Krise geraten, durch die teilweise durch Turbulenzen hindurch die Fähigkeiten erworben werden, mit den neu sich stellenden und immer komplexer werdenden Herausforderungen und Aufgaben fertig zu werden (Peukert, 2015, S. 24f., S. 58f., S. 145-147, S. 308-310). 
Dieser Prozess kann „,von außen“ nicht gesteuert, sehr wohl aber unterstützt und gefördert werden, indem die Heranwachsenden von den Erwachsenen von Anfang an als Ko-Konstrukteure ihrer Entwicklung anerkannt und ernst genommen und ihnen die Handlungsspielräume eröffnet werden, die sie benötigen, um je ein neues Verhältnis zu sich und der sie umgebenden Wirklichkeit gewinnen zu können. Dazu gehören ein Lernen in Form eines voranschreitenden Erwerbs vorliegender Wissensbestände, aber auch die Offenheit und der Mut dazu, wo dieses Wissen angesichts neu sich stellender Herausforderungen und Aufgaben nicht hinreicht, „,bewußt neue Weisen der Wahrnehmung von Wirklichkeit und des Umgangs mit Sachen, Personen und sich selbst zu erarbeiten, also auch eine neue Identität zu finden" (Peukert, 2015, S. 259). Auch für die Erziehenden können sich bei ihrer Begleitung der Heranwachsenden neue Welten auftun. So wichtig es ist, im Nahbereich solche Erfahrungen im Umgang miteinander machen zu können, so unerlässlich ist es, soll dieser geschützt werden, geltend zu machen und sich dafür einzusetzen, dass in der Gesellschaft insgesamt die Möglichkeit zu gemeinsamer Selbstbestimmung und der Gestaltung eines solidarischen Lebens auf Zukunft hin gewährleistet und institutionell abgesichert ist. Dazu gehört nicht zuletzt die Einrichtung von Bildungsinstitutionen, in denen die Fähigkeiten dazu erworben und eingeübt werden können. Die Verständigung über eine zukunftsfähige Gestaltung der Gesellschaft ist allerdings eine Aufgabe, die alle angeht sowie von und in allen Lebensbereichen und Handlungssystem, gerade auch dem wirtschaftlichen, bewerkstelligt werden muss. Die im pädagogischen Bereich Tätigen sind aufgrund ihres direkten Umgangs mit den kommenden Generationen in besonderer Weise dafür sensibilisiert und haben eine entsprechende Verantwortung wahrzunehmen.

Wenn diese Reflexionen in zutreffender Weise bestimmen, was pädagogisches Handeln der Struktur nach leitet, dass ihm also konstitutiv eine transformatorische Struktur innewohnt, dann könnte sich das nach Peukert "gerade unter der Erfahrung, daß moderne Gesellschaften eine Dynamik entfalten, die einerseits mehr Menschen als je zuvor Leben ermöglicht, zugleich aber ihre Lebensgrundlagen bedroht [...] sowohl als Prinzip von Bildung wie als Prinzip einer humanen Gesellschaft erweisen: In endlicher Freiheit so zu existieren, daß man versucht, im eigenen produktiven Handeln anderen zu eigenständiger, kreativer Entfaltung zu verhelfen und so eine gemeinsame kommunikative Welt aufzubauen, in der Regeln für das Zusammenleben aller gefunden und dadurch Lebensmöglichkeiten für alle unter einer transformationsfähigen demokratischen ,Verfaßtheit' der Gesellschaft gesichert werden können“ (Peukert, 2015, S. 43).

Maureen Junker-Kenny hebt die aktuelle Brisanz einer solchen Bestimmung von pädagogischem Handeln hervor, wenn sie schreibt: „Intersubjektive Kreativität als Programm der Bildung ist eine Überlebensfrage spätmoderner, in ihrer ,Entwickeltheit' sich selbst überschlagender Gesellschaften“ (JunkerKenny, 2010, S. 60).

Das impliziert, dass nicht am einsamen Schreibtisch Deutungs- und Orientierungsmuster erfunden werden können, mit denen die nächsten Generationen die sich ihnen stellenden Herausforderungen und Aufgaben meistern können, sondern dass diese nur gemeinsam mit ihnen zu finden sind (Peukert, 2015, S. 333f.). Dabei sind kein Lebensbereich und kein gesellschaftlicher Sektor von einer kritischen Überprüfung auf ihre Zukunftstauglichkeit hin ausgenommen. Insgesamt steht angesichts der gerade für die nachkommenden Generationen desolaten Lage nichts weniger an als ein ethischer Bewusstseinssprung. Das heißt nicht, die Vergangenheit einfach hinter sich zu lassen. In seinem Durchgang durch das als klassisch zu würdigende Erziehungs- und Bildungsdenken und in der Rückbesinnung auf die noch nicht eingelösten Verheißungen aus vergangenen Zeiten zeigt Peukert, welche produktiven und innovativen Kräfte sie in sich bergen, um die anstehenden Transformationen anzugehen.

\section{Kritische Bewusstseinsbildung (Paulo Freire)}

„Die Kosten der Entwicklung moderner Gesellschaften haben gegenwärtig vor allem die Länder der so- 
genannten dritten und vierten Welt zu tragen. Sie werden nicht nur ärmer durch den Abzug von Kapital und Ressourcen bei gleichzeitigem Wachstum der Bevölkerung; die politische Unterdrückung und die ökonomische Ausbeutung führen auch zur psychischen Verelendung und Desintegration, zur Zunahme von Gewalt und weiterer Brutalisierung politischer Herrschaft, unter der menschliches Leben entwertet wird. Freire hat sich in Situationen extremster Armut und Unterdrückung begeben. Sinn seiner Alphabetisierungskampagnen war, auch in solchen Situationen noch die Möglichkeit von Freiheit und Selbstbestimmung wahrzunehmen. Pädagogisches Handeln versucht dabei Hilfe zu leisten, die eigene Situation in ihrer Widersprüchlichkeit zu buchstabieren, dabei sich selbst artikulieren zu lernen und gemeinsame Handlungsmöglichkeiten zu erschließen, und zwar ohne das Bewußtsein der Unterdrücker zu übernehmen, sie also noch einmal über sich siegen zu lassen und den Zirkel der Gewalt fortzusetzen“ (Peukert, 1988, S. 185).

So hat Helmut Peukert die 1970 erschienene „Pädagogik der Unterdrückten“, im chilenischen Exil verfasst vom brasilianischen Pädagogen Paulo Freire (1921-1997), knapp zusammengefasst und gewürdigt. Zeit seines Schaffens war Paulo Freire von der Überzeugung beseelt, dass eine andere Welt als die bestehende möglich ist - eine Überwindung der bestehenden Welt, in der ein Teil - genauer: der kleinere Teil - der Menschheit das Leben in vollen Zügen genießen könne, während der größere Teil der Weltbevölkerung ihre Existenz in Armut und Elend fristen müsse. Die Aufgabe von Erziehung und Bildung sah er darin, zur Schaffung einer solchen neuen Welt beizutragen (zum Folgenden ausführlicher Mette, 2020).

Freires anthropologischer Grundannahme zufolge ist das menschliche Sein nicht a priori gegeben, sondern radikal unabgeschlossen und somit offen für Neues. Es gelte deshalb, die menschliche Neugier zu entfachen, indem die Menschen sich ihrer Unvollendetheit bewusst würden. Die Berufung des Menschen sei es, immer mehr zu dem zu finden, was ihn in seinem Wesen ausmache, zu seiner Freiheit bzw. Autonomie, die ihn befähige, verantwortlich seine eigenen Geschicke in die Hand zu nehmen und an der Auferbauung einer Welt mitzuwirken, die allen Menschen ein Leben in ihrer unantastbaren Würde und unter Inanspruchnahme ihrer unveräußerlichen Rechte ermöglicht. In dieser Bildungsfähigkeit hat nach Freire die Hoffnung als genuin menschliche Tugend ihren Grund. Sie räume den Träumen ihre Berechtigung ein, aus denen ihrerseits Utopien und Visionen eines guten Lebens für alle erwachsen.

Mit seiner Einwurzelung in die konkrete Geschichte hängt die Bestimmung des Menschen als gemeinschaftliches Wesen zusammen. Bildung ist nach Freire von Grund auf ein sozialer Prozess, erfolgt in Interaktion und Kommunikation zwischen Subjekten, die untereinander - so bezeichnet es Freire - in "dialogischer Beziehung" stehen. Im Austausch der jeweiligen Weltsichten, die die Einzelnen mitbringen, und in deren gemeinsamer kritischer Prüfung erfolgt eine neue soziale Konstruktion der Wirklichkeit. Das dialogisch-solidarische Miteinander ist unabdingbare Voraussetzung für den in Freiheit führenden Exodus aus ungerechten Verhältnissen. Insofern haftet dem Bildungsprozess nach Freire etwas an, was als basisdemokratisch bezeichnet werden kann und politisch im weiteren Sinne ist. Dabei war Freire sich bewusst, dass durch Erziehung allein keine neue Welt geschaffen werden kann. Aber sich als Teil der sozialen Bewegung verstehend, die vor Ort und weltweit gegen Armut und Hunger, Unterdrückung, Ausbeutung und Exklusion kämpft, kann sie ihren Beitrag zur Schaffung einer Welt einbringen, die ein Leben in Würde für alle Menschen bereit hält.

Nach Freire hat die Bildungsarbeit (Alphabetisierung) damit zu beginnen, dass an den Lebenswelten der Leute angeknüpft wird und ihre Weltsicht bzw. Einstellung und das darin aufbewahrte Wissen (Weisheit) aufgegriffen werden, um die Lernenden dann in ein reflexives Verhältnis dazu zu bringen und sie entdecken zu lassen, von woher dieses ihr Wissen geprägt worden ist und welche Elemente daraus ihnen zu ihrer Subjektwerdung verhelfen und welche sie verhindern. Dieses mündet schließlich in das Bestreben, sich gemeinsam mit den anderen ähnlich Betroffenen von den Hindernissen zu befreien. 
Lernen und Sich-Bilden besteht nicht in der Akkumulation von Wissen. Denn das laufe letztlich auf eine Anpassung der Lernenden auf den Status quo hinaus. Dagegen setzt Freire die problematisierende Methode, mithilfe derer es darum zu tun ist, dass die Lernenden - gemeinsam mit der Lehrkraft - das ihnen vorgesetzte Wissen nicht einfach übernehmen, sondern es kritisch befragen, auf die zugrundeliegenden Annahmen hin prüfen, es in Zweifel ziehen, aber auch das, was der Prüfung standhält, gelten lassen und so ein kritisches Bewusstsein gegenüber der Wirklichkeit ausbilden. So vollziehen sich Lehren, Lernen und Sich-Bilden für alle Beteiligten als ein transformativer Prozess, aus dem sie anders hervorgehen, als sie in ihn hineingegangen sind - und zwar im Sinne einer individuellen und kollektiven Transformation in Richtung größerer Humanität.

So sehr bei dem Übergang von einem naiven zu einem kritischen Bewusstsein die Vernunft eine Rolle spielt, so will Freire diesen Vorgang nicht auf diese rationale Dimension begrenzt wissen. Es handelt sich vielmehr um ein ganzheitliches Projekt, in dem Vernunft, Sinnesempfindungen, Gefühle und Wünsche, Geist und Körper eine Einheit bilden. Es wird motiviert und vorangetrieben durch die Hoffnung, ergänzt - wie Freire es ebenfalls später stark hervorhebt - durch die Freude, durch die Freude am eigenen Leben und an der Welt. Diese Freude auszustrahlen und weiterzugeben, mache eine zentrale Eigenschaft derer aus, die im Bereich von Erziehung und Bildung tätig sind.

Ein weiteres Element in Freires Werk, das in engem Zusammenhang mit den bisher genannten steht, ist die Ethik. Es gibt, so schreibt er, ,keine Möglichkeit humaner Bildung ohne Ethik. [...] Die ethischen Erfordernisse werden kritischer in einer Welt, die immer weniger ethisch wird" (Freire, 2007, S. 134). Die ethische Haltung erwächst nach ihm aus der Empörung darüber, dass die Mehrheit der Menschen auf dieser Erde ihr Leben unter Bedingungen fristen muss, die ihnen ihre Würde und ihre Rechte vorenthalten, und dass damit die Welt nicht so ist, wie sie sein könnte. Auch ihr ist somit ein Drang zur Transformation der gegebenen Verhältnisse zu Eigen. „Indem wir fähig werden, die Welt zu begreifen“, schreibt Paulo Freire, „das Begriffene zu kommunizieren, zu beobachten, zu vergleichen, zu entscheiden, mit Dingen zu brechen, zu wählen, zu benoten, werden wir zu ethisch orientierten Wesen“ (Freire, 2007, S. 103).

Dieses Verständnis von Ethik sah Freire aktuell im höchsten Maße gefährdet, wenn nicht verdrängt durch eine von ihm sog. „Ethik des Marktes“. Sie dulde keine Veränderungen zum Besseren. „Die Ethik des Gewinns, unter dessen Interessen wir Frauen und Männer uns unterwerfen müssen, steht den [...] humanen Interessen völlig entgegen: die Reichen und Herrschenden genießen, die Armen und Unterdrückten leiden“ (Freire, 2007, S. 98).

Hinter dieser sich mehr und mehr ausbreitenden Ethik des Marktes steckt nach Freire die Ideologie des Neoliberalismus, die im Zuge der von ihm gesteuerten Globalisierung die Weltherrschaft angetreten hat. Diese Ideologie drohe „unser Gehirn zu betäuben, die Neugierde zu verwirren, die Wahrnehmung von Fakten, Angelegenheiten und Geschehnissen zu verzerren" (Freire, 2008, S. 120), indem sie das Ende der Geschichte proklamiere und jeden Traum und jede Utopie töte, indem sie darauf insistiere, dass es keine Alternative zum Bestehenden gebe. „Wenn der Traum stirbt", so argumentiert Freire, "und die Utopie auch, verfügt die erzieherische Praxis über nichts mehr, um die verbrecherische Realität anzuklagen und eine weniger schlechte, humanere Realität anzukündigen. Es kommt zum Konzept von Erziehung als einer rigoros pragmatischen Praxis, die zu Erziehenden sind in der Anwendung wissenschaftlicher Techniken und Prinzipien zu trainieren. Der neoliberale Pragmatismus birgt nichts in sich, was nach Bildung aussieht" (Freire, 2007, S. 104). Einer solchen funktionalen Reduktion pädagogischen Handelns auf Schulung und Training der Kompetenzen, die im Interesse des herrschenden Systems liegen - ohne Rücksicht auf die damit einhergehende Spaltung der Gesellschaft in diejenigen, die gebraucht werden, und diejenigen, die nicht gebraucht werden -, galt Freires entschiedener und rebellischer Widerspruch. 
„Ich kann nicht schweigend hinnehmen“, so notierte er kurz vor seinem Tode, „und mit ,gutem Benehmen' hinnehmen, dass eine Milliarde von Arbeitslosen, mit denen dieses Jahrhundert abschließt, als pures Schicksal dieses Moments betrachtet wird. Keine soziale, historische, wirtschaftliche Realität ist so, weil geschrieben wird, dass es so sei. Solange ich in der Geschichte und in der Welt präsent bin, kämpfe ich voller Hoffnung für den Traum, für die Utopie, für die Hoffnung in der Perspektive einer Kritischen Pädagogik. Dies ist kein leerer, kein vergeblicher Kampf" (Freire, 2007, S. 121).

\section{Ein neuer Bildungspakt (Papst Franziskus)}

Papst Franziskus (*1936) zählt gegenwärtig zu den Persönlichkeiten an führender Stelle in einer weltweit verbreiteten Institution, der alles in seinen Kräften Mögliche dafür tut, der Menschheit einen Spiegel vor Augen zu halten, in dem sich ungeschminkt die krisenhafte Lage, in der sie sich befindet, widerspiegelt, um gemeinsam mit höchster Dringlichkeit nach Wegen eines gerechten, friedlichen und nachhaltigen Miteinanders zu suchen. In seinen großen Texten (Papst Franziskus, 2013; 2015; 2020a; 2020b) und zusätzlich in vielen Botschaften und Ansprachen fordert er beharrlich auf, um der Ermöglichung eines gemeinsamen Lebens aller auf Zukunft hin endlich eine gerechte und solidarische Wirtschaftsordnung zu installieren, sich nicht gegen die Menschen auf der Flucht abzuschließen, den Raubbau der Natur zu unterlassen, das mörderische Wettrüsten zu beenden und konsequent militärisch abzurüsten, eine Kultur der Liebe und Wahrheit zu schaffen, den Dialog und die Kooperation zwischen den Religionen zu forcieren und vieles andere mehr. Immer wieder kommt er dabei auch auf die Bedeutung der Erziehung und Bildung in diesem Zusammenhang zu sprechen. Um dem noch ein stärkeres Gewicht zu verleihen und konkrete Projekte zu initiieren, hat er im September 2019 zum Start eines Bildungspakts eingeladen (Papst Franziskus, 2019). Auf welcher Grundlage dieser Pakt arbeiten soll, an dem mitzuwirken nicht nur die im Erziehungs- und Bildungsbereich tätigen Erwachsenen und Heranwachsenden eingeladen sind, sondern alle weltweit in Kultur, Wissenschaft, Kunst, Medien und Sport Tätigen, hat er in seiner Videobotschaft an ein virtuelles Treffen im Jahr 2020 wie folgt dargelegt:

„Erziehen heißt darauf zu setzen und der Gegenwart eben diese Hoffnung zu geben, welche die deterministischen und fatalistischen Theorien durchbricht, mit denen sich der Egoismus der Starken, der Konformismus der Schwachen und die Ideologie der Utopisten oft als angeblich einzig möglicher Weg aufdrängen. Erziehen ist immer ein Akt der Hoffnung; er ruft zur Mitbeteiligung auf und zur Umwandlung der sterilen, lähmenden Logik der Gleichgültigkeit in ein anderes Denken, das unsere gegenseitige Zugehörigkeit berücksichtigt. Wenn sich heute die Bildungssysteme nach einer Logik der Ersetzbarkeit und Wiederholung richten und es nicht vermögen, neue Horizonte zu entwickeln und aufzuzeigen, in denen Gastfreundschaft, Generationensolidarität und der Wert der Transzendenz eine neue Kultur begründen, verpassen wir dann nicht eine historische Gelegenheit? Wir sind uns auch bewusst, dass Lebenswege eine Hoffnung brauchen, die auf Solidarität gründet. Jede Veränderung erfordert einen Bildungsprozess, um neue Paradigmen herauszubilden, die auf die Herausforderungen und Notlagen der heutigen Welt reagieren, die Bedürfnisse der verschiedenen Generationen verstehen und Lösungen für sie finden und die Menschheit von heute und morgen zum Blühen bringen. [...] Bildung bietet sich daher als das natürliche Gegenmittel zur individualistischen Kultur an, die bisweilen in einen wahren Kult des Ich und in die Vorherrschaft der Gleichgültigkeit ausartet. Unsere Zukunft darf nicht von der Spaltung, von der Verarmung des Denkens und der Vorstellungskraft, des Zuhörens, des Dialogs und des gegenseitigen Verständnisses gekennzeichnet sein. Das darf nicht unsere Zukunft sein. [...] Eine andere Welt ist möglich und erfordert, dass wir sie aufzubauen lernen, und das betrifft unser ganzes Menschsein, sowohl auf persönlicher als auch auf gemeinschaftlicher Ebene" (Papst Franziskus, 2020c).

Vor Überforderung und Resignation wird dieses Engagement bewahrt, so betont der Papst, wenn es von einer tief in der Existenz verwurzelten Spiritualität getragen wird.

Als beispielhaft für eine von einer Ethik universaler Solidarität unterlegte politische Bildungsarbeit 
führt er das Engagement der Sozialen Bewegungen an (Kern, 2018). Er hat sie als „,soziale Poeten“ charakterisiert, weil sie der Ungerechtigkeit nicht das letzte Wort ließen, sondern sie in neue Möglichkeiten verwandeln würden (Papst Franziskus, 2020b, S. 154). Auf der Videokonferenz mit den Sozialen Bewegungen im Oktober 2020 wurde vorgeschlagen, das Projekt der Gründung einer „Universidad de las Periferias" in Angriff zu nehmen.

\section{Literaturverzeichnis}

Bederna, Katrin (2019). Every Day for Future. Theologie und religiöse Bildung für nachhaltige Entwicklung. Ostfildern: Matthias Grünewald.

Benjamin, Walter (1991). Charles Baudelaire. Ein Lyriker im Zeitalter des Hochkapitalismus. In Walter Benjamin, Gesammelte Schriften, Band 1 (S. 654-690). Frankfurt Main: Suhrkamp.

Freire, Paulo (2007). Bildung und Hoffnung. Münster: Waxmann.

Freire, Paulo (2008). Pädagogik der Autonomie. Münster: Waxmann.

Gärtner, Claudia (2020). Klima, Corona und das Christentum. Religiöse Bildung für nachhaltige Entwicklung in einer verwundeten Welt. Bielefeld: transcript.

Junker-Kenny, Maureen (2010). Der Gipfel des Schöpferischen. Das Jesuszeugnis als Quelle öffentlicher Vernunft. In Ottmar John \& Magnus Striet (Hg.), "... und nichts Menschliches ist mir fremd": theologische Grenzgänge (S. 59-75). Regensburg: Pustet.

Kern, Benedikt (2018). Radikal Welt verändern. Papst Franziskus und die Sozialen Bewegungen. Münster: Edition ITP-Kompass.

Lang-Wojtasik, Gregor \& Erichsen-Morgenstern, Ronja M. (2019). Transformation als Herausforderung. Globales Lernen als lebenslanger Bildungsauftrag für alle. In Walter Leal Filho (Hg.), Aktuelle Ansätze zur Umsetzung der UN-Nachhaltigkeitsziele, Band 1 (S. 251-270). Berlin: Springer Spektrum.

Mette, Norbert (2020). Hoffnung - Universale Ethik - Autonomie. Zentrale Begriffe und Linien im späteren Werk von Paulo Freire. Dialogische Erziehung, 24(1-2), 52-62.

Österreichisches Religionspädagogisches Forum [ÖRF] (2020). Themenheft Nachhaltiges Lernen. ÖRF 28(2).

Papst Franziskus (2013). Apostolisches Schreiben Evangelii gaudium des Heiligen Vaters Papst Franziskus. Bonn: Sekretariat der Deutschen Bischofskonferenz.

Papst Franziskus (2015). Enzyklika Laudato si' von Papst Franziskus über die Sorge für das gemeinsame Haus. Bonn: Sekretariat der Deutschen Bischofskonferenz.

Papst Franziskus (2019). Botschaft zum Start des Bildungspakts am 12.09.2019. Abgerufen von https://www.vatican.va/content/francesco/de/messages/pont-messages/2019/documents/papa-francesco 20190912 messaggio-patto-educativo.html [03.02.2021]

Papst Franziskus (2020a). Enzyklika Fratelli tutti von Papst Franziskus über die Geschwisterlichkeit und die soziale Freundschaft. Bonn: Sekretariat der Deutschen Bischofskonferenz

Papst Franziskus (2020b). Wage zu träumen! Mit Zuversicht aus der Krise. München: Kösel.

Papst Franziskus (2020c). Videobotschaft aus Anlass der Begegnung "Global Compact on Education. Together to Look Beyond" am 15. Oktober 2020. Abgerufen von http://www.vatican.va/content/francesco/de/messages/pont-messages/2020/documents/papa-francesco 20201015 videomessaggio-globalcompact.html [03.02.2021]

Peukert, Helmut (1988). Praxis universaler Solidarität. Grenzprobleme im Verhältnis von Erziehungswissenschaft und Theologie. In Edward Schillebeeckx (Hg.), Mystik und Politik. Theologie im Ringen um Geschichte und Gesellschaft (S. 172-185). Mainz: Matthias Grünewald.

Peukert, Helmut (2009). Wissenschaftstheorie - Handlungstheorie - Fundamentale Theologie. Analysen zu Ansatz und Status theologischer Theoriebildung (3. Auflage mit einem Vorwort zur Neuauflage). Frankfurt am Main: Suhrkamp. 
Peukert, Helmut (2015). Bildung in gesellschaftlicher Transformation. Paderborn: Schöningh.

Seitz, Klaus (2017). Transformation als Lernprozess und Bildungsaufgabe. In Oliver Emde, Uwe Jakubczyk, Bernd Kappes \& Bernd Overwien (Hg.), Mit Bildung die Welt verändern? Globales Lernen für eine nachhaltige Entwicklung (S. 160-168). Opladen: Barbara Budrich.

Scheunpflug, Annette (2019). Transformatives Globales Lernen - eine Grundlegung in didaktischer Absicht. In Gregor Lang-Wojtasik (Hg.), Bildung für eine Welt in Transformation: Global Citizenship Education als Chance für die Weltgesellschaft (S. 63-74). Opladen: Barbara Budrich.

Wissenschaftlicher Beirat Globale Umweltveränderungen [WBGU] (2011). Welt im Wandel. Gesellschaftsvertrag für eine Große Transformation (2. Auflage). Berlin: WBGU. 